\title{
Possible Tetraquark Explanation for the Proposed Tcc+
}

Joseph Bevelacqua

Funding: The author(s) received no specific funding for this work.

Potential competing interests: The author(s) declared that no potential competing interests exist.

\section{Abstract}

The recently proposed $\mathrm{T}_{\mathrm{Cc}}{ }^{+}$structure is investigated using a first-order tetraquark mass formula. This mass relationship is based on weakly bound $D^{*+}(c d-b a r)+D^{0}(c)$-bar $)$ meson clusters and provides a reasonable prediction (within about $1.4 \%$ ) of the measured $\mathrm{T}_{\mathrm{Cc}}{ }^{+}$mass.

\subsection{Introduction}

CERN's LHCb experiment presented the discovery of a new tetraquark at the 2021 European Physical Society Conference on High Energy Physics (EPS-HEP) ${ }^{1}$. The new particle, labeled as the $\mathrm{T}_{\mathrm{CC}}{ }^{+}$is comprised of two c quarks and $u$ and $d$ antiquarks ${ }^{1}$. The tetraquark occurs as a narrow peak in the $D^{0} D^{0} \pi^{+}$meson mass spectrum, just below the $D^{0}$ (c u-bar) + $D^{*+}$ (c d-bar) mass threshold, with a statistical significance exceeding 20 standard deviations ${ }^{1}$. The $\mathrm{T}_{\mathrm{Cc}}{ }^{+}$is the longest-lived exotic matter particle discovered to date based on its width of about $400 \mathrm{keV}^{1}$. As noted in Fig. 1 of Ref. 1, the mass is about $3875 \mathrm{MeV} / \mathrm{c}^{2}$, but no spin-parity values are provided.

Based on Ref. 1 , the $\mathrm{T}_{\mathrm{CC}}{ }^{+}$tetraquark candidate would couple to the $\mathrm{D}^{0}+\mathrm{D}^{*+}$ channel. The $\mathrm{T}_{\mathrm{Cc}}{ }^{+}$tetraquark has unit positive charge with a most likely c u-bar c d-bar quark configuration.

In this paper, the first-order tetraquark mass formulas of Refs. 2 - 9 are applied to evaluate the possible configuration of the $\mathrm{T}_{\mathrm{Cc}}{ }^{+}$state. The first-order model is utilized to predict both the mass and ${ }^{\pi}$ value of the $\mathrm{T}_{\mathrm{cc}}{ }^{+}$ tetraquark.

\subsection{Model and Formulation}

Zel'dovich and Sakharov ${ }^{10,11}$ proposed a semiempirical mass formula that provides a prediction of mesons and baryons in terms of effective quark masses. Within this formulation, quark wave functions are assumed to reside in their lowest $1 \mathrm{~S}$ state. These meson mass formulas are used as the basis for deriving a first-order tetraquark mass formula. In particular, the model proposed in this paper assumes the tetraquark is partitioned into two clusters with the interaction between the clusters providing a minimal contribution to the tetraquark mass.

The meson mass (M) formula of Refs. 2 and 3 is:

$$
M_{m}=\delta_{m}+m_{1}+m_{2}+b_{m}\left[m_{0}{ }^{2} /\left(m_{1} m_{2}\right)\right] \sigma_{1} \cdot \sigma_{2}
$$

where $m_{1}\left(m_{2}\right)$ are the mass of the first (second) quark comprising the meson, $m_{0}$ is the average mass of a first generation quark ${ }^{10,11}$, and the $\sigma_{i}(i=1$ and 2$)$ are the spin vectors for the quarks incorporated into the meson. 
The parameters $\delta_{m}$ and $b_{m}$ are $40 \mathrm{MeV} / \mathrm{c}^{2}$ and $615 \mathrm{MeV} / \mathrm{c}^{2}$, respectively ${ }^{11}$.

The last term in Eq. 1 represents the spin-spin interaction of the quarks and $\sigma_{1} \cdot \sigma_{2}$ is the scalar product of the quark spin vectors. $\boldsymbol{\sigma}_{\mathbf{1}} \cdot \boldsymbol{\sigma}_{\mathbf{2}}$ has the value $-3 / 4$ and $+1 / 4$ for pseudoscalar and vector mesons, respectively ${ }^{11}$.

In formulating the tetraquark mass formula, effective quark masses provided by Griffiths ${ }^{12}$ are utilized. These effective masses for $d, u, s, c, b$, and t quarks are 340, 336, 486, 1550, 4730, and $177000 \mathrm{MeV} / \mathrm{c}^{2}$, respectively. These masses are utilized in Eq. 1.

These six quarks are arranged in three generations: $[d(-1 / 3), u(+2 / 3)],[s(-1 / 3), c(+2 / 3)]$, and $[b(-1 / 3)$, $t(+2 / 3)]^{13}$. The three generations are specified by the square brackets and the quark charges are given within parentheses.

\subsection{First-Order Mass Formula for the $\mathrm{T}_{\mathrm{cc}}{ }^{+}$}

The spin of a tetraquark within the first order mass formula is determined by coupling the two meson clusters

$J^{\pi}=J^{\pi}(1) \times L \times J^{\pi}(2)$

where the first-order mass formula assumes a minimally interacting $L=0$ configuration ${ }^{2,3}$ between the meson clusters. No $\mathrm{J}^{\pi}$ assignment was provided in Ref. 1. Eq. 2 provides a $\mathrm{J}^{\pi}$ assignment using the possible meson clusters noted in Ref. 1 . These include the $D^{0}$ and $D^{*+}$ with $J^{\pi}$ values of $0^{-}$and $1^{-}$, respectively ${ }^{13}$. The first-order mass formula $\mathrm{J}^{\pi}=1^{+}$assignment ${ }^{1}$ for the $\mathrm{T}_{\mathrm{cc}}{ }^{+}$follows naturally from the $0^{-} \times 0 \times 1^{-}$coupling structure of Eq. 2 .

The first-order mass formula used in this paper partitions the tetraquark into two meson clusters. The first cluster is a $D^{0}$ scalar meson $(\mathrm{sm})$ and the remaining cluster is a $D^{*+}$ vector meson (vm). These simplifications are incorporated to minimize model complexity which is consistent with an initial first-order formulation. In addition, the general tetraquark mass formula is assumed to have the form ${ }^{2,3}$

$\mathbf{M}=\mathbf{M}_{\mathrm{sm}}+\mathbf{M}_{\mathrm{vm}}+\boldsymbol{\Phi}$

where $\Phi$ defines the interaction between the meson clusters. Within the scope of this mass formula, the mesonmeson cluster interaction is assumed to be weak and sufficiently small to be ignored. Accordingly, Eq. 3 represents a quasimolecular four quark system whose basic character is a weakly bound meson-meson system.

\section{1 $\mathrm{D}^{0}+\mathrm{D}^{*+}$ Meson Clusters}

In the $D^{0}+D^{*+}$ configuration, the scalar meson is $D^{0}$ and the vector meson is $\mathrm{D}^{*+}$. For the $\mathrm{T}_{\mathrm{cc}}{ }^{+}$, the vector meson is the $\mathrm{D}^{*+}$ and not the $\mathrm{D}^{+}$ground state. Since the first-order $\mathrm{T}_{\mathrm{cc}}{ }^{+}$tetraquark mass formula involves an excited $D^{*+}$ meson cluster, the mass formula is modified to account for this excitation energy $\mathrm{y}^{2,3}$ :

$\mathbf{M}=\mathbf{M}_{\mathrm{sm}}+\mathbf{M}_{\mathrm{vm}}{ }^{*}+\Phi$

where

$\mathbf{M}_{\mathrm{vm}}{ }^{*}=\mathbf{M}_{\mathrm{vm}}+\Delta\left(\mathbf{D}^{*+}-\mathrm{D}^{+}\right)$

and $\Delta$ is the $D^{*+}-D^{+}$energy difference $\left(140.61 \mathrm{MeV} / \mathrm{C}^{2}\right)^{13}$.

\subsection{Results and Discussion}

The angular momentum coupling from Eq. 2 and the first-order mass formula of Eqs. 1 and 3-5 are used to construct a $\mathrm{T}_{\mathrm{cc}}{ }^{+}$state. This state is modeled as noted in Sections 3.1.

The first-order mass formula provides a reasonable representation of the $\mathrm{T}_{\mathrm{CC}}{ }^{+}$tetraquark structure. The $\mathrm{D}^{0}+$ 
$\mathrm{D}^{*+}$ configuration results in a mass of $3928.76 \mathrm{MeV} / \mathrm{C}^{2}$ that is that is about $1.4 \%$ larger than the $3875 \mathrm{MeV} / \mathrm{C}^{2}$ experimental value ${ }^{1}$.

Although these results are encouraging, they are based on a first-order mass formula with a number of uncertainties including the assumed quark masses ${ }^{12}$ and the magnitude of the meson-meson cluster interaction. However, the model does provide an initial description of the $\mathrm{T}_{\mathrm{cc}}{ }^{+}$mass and provides a $\mathrm{J}^{\pi}=1^{+}$ assignment in terms of a tetraquark structure.

\subsection{Conclusions}

The first-order mass formula predicts $D^{0}+D^{*+}$ configuration for the $T_{c c}{ }^{+}$. Weakly bound $D^{0}+D^{*+}$ meson clusters lead to a predicted mass that is within about $1.4 \%$ of the measured value ${ }^{1}$. Although Ref. 1 does not provide a $\mathrm{J}^{\pi}$ assignment for the $\mathrm{T}_{\mathrm{Cc}}{ }^{+}$, the model suggests a $\mathrm{J}^{\pi}=1^{+}$assignment derived from Eq. 2 .

\section{References}

1) European Physical Society conference on high energy physics, EPS-HEP 202129 July 2021: Observation of an exceptionally charming tetraquark. Results provided in https://lhcb-public.web.cern.ch/Welcome.htm/\#Tcc and //indico.desy.de/event/28202/contributions/105627/attachments/67806/84639/EPS-HEP_2021_Polyakov_v5.pdf.

2) J. J. Bevelacqua, First-Order Tetraquark Mass Formula, Physics Essays 29, 198 (2016).

3) J. J. Bevelacqua, Description of the X(5568) and Proposed $750 \mathrm{GeV} / \mathrm{c}^{2}$ State in Terms of a First-Order Tetraquark Mass Formula, Physics Essays 29, 367 (2016).

4) J. J. Bevelacqua, Fusion of doubly heavy mesons into a tetraquark, Physics Essays 31, 167 (2018).

5) J. J. Bevelacqua, Possible Tetraquark Explanation for the Proposed X(3872), Physics Essays 32, 469 (2019).

6) J. J. Bevelacqua, Description of the $X(6900)$ as a Four Charmed Quark State in Terms of a First-Order Tetraquark Mass Formula, QEIOS KLXLKJ, 1 (2020).

https://doi.org/10.32388/KLXLKJ.

7) J. J. Bevelacqua, Description of the $X(2900)$ as an Open Flavor Tetraquark in Terms of a First-Order Mass Formula, QEIOS, OVLMEB, 1 (2020).

https://doi.org/10.32388/OVLMEB.

8) J. J. Bevelacqua, Possible Tetraquark Explanation for the Proposed $Z_{C S}(3985)^{-}$, QEIOS

GLTEU2, 1 (2021). https://doi.org/10.32388/GLTEU2.

9) J. J. Bevelacqua, Possible Tetraquark Explanation for the X(6200), QEIOS J6AFYW, 1 (2021). https://doi.org/10.32388//6AFYW.

10) Ya. B. Zel'dovich and A. D. Sakharov, Kvarkovaia struktura i massy sil'novzaimodeistvuyushchikh chastits, Yad. Fiz. 4, 395 (1966).

11) A. D. Sakharov, Mass formula for mesons and baryons, Sov. Phys. JETP 51, 1059 (1980).

12) D. Griffiths, Introduction to Elementary Particles, $2^{\text {nd }}$ ed., (Wiley-VCH, Weinheim, 2008).

13) Particle Data Group, Review of Particle Physics, Prog. Theor. Exp. Phys. 2020, $083 C 01$ (2020). 
